\title{
Levels of Blood Biomarkers among Patients with Myocardial Infarction in Comparison to Control Group
}

Amir Shamshirian 1,2,9, Reza Alizadeh-Navaei ${ }^{2}$,Samira Abedi ${ }^{3}$, Hamed Jafarpour ${ }^{3}$, Hanieh Fazli $^{3}$, Samira Hosseini ${ }^{1}$, Amirhossein Hessami ${ }^{3}$, Keyvan Karimifar ${ }^{4}$, Sedighe Yosefi ${ }^{5}$, Mohammad Zahedi ${ }^{1}$, Sepideh Motamen ${ }^{6}$, Atiyeh Ghorbanpour ${ }^{7}$, Bahman Zarandi ${ }^{7}$, Aliakbar Esfahani $^{4}$, Yeganeh Rostamian-Moghaddam ${ }^{8}$, Shirin Mehdipour ${ }^{9}$, Keyvan Heydari ${ }^{3}$, Sedigheh Aghajanian', Somayeh Pour Mehdi', Alireza Azad ${ }^{9}$, Soheil Azizi1,9*

\footnotetext{
OPEN ACCESS

Citation: Amir Shamshirian, Reza AlizadehNavaei, Samira Abedi. Levels of Blood Biomarkers among Patients with Myocardial Infarction in Comparison to Control Group. Ethiop J Health Sci. 2020;30(1):5.doi:http://dx.doi.org/10.4314/ejhs.v30 i1.2

Received: July 08, 2018

Accepted: July 24, 2019

Published: January 1, 2020

Copyright: (C2020 Shamshirian A, et al. This is an open access article distributed under the terms of the Creative Commons Attribution License, which permits unrestricted use, distribution, and reproduction in any medium, provided the original author and source are credited.

Funding: Student Research Committee, Mazandaran University of Medical Sciences

Competing Interests: The authors declare that this manuscript was approved by all authors in its form and that no competing interest exists.

Affiliation and Correspondence:

${ }^{1}$ Department of Medical Laboratory Sciences,

School of Allied Medical sciences, Mazandaran

University of Medical Sciences, Iran.

${ }^{2}$ Gastrointestinal Cancer Research Center, Mazandaran University of Medical Sciences, Iran.

${ }^{3}$ Student Research Committee, School of

Medicine, Mazandaran University of Medical

Sciences, Iran.

${ }^{4}$ Student Research committee, Mashhad University of Medical Sciences, Mashhad, Iran.

${ }^{5}$ Department of Biochemistry, Faculty of

Medicine, Semnan University of Medical

Sciences, Semnan, Iran.

${ }^{6}$ Department of Medical Biotechnology, Faculty of Medical Sciences, Tarbiat Modares University, Iran.

${ }^{7}$ Department of Hematology, Student Research Committee, Iran University of Medical Sciences, Iran.

${ }^{8}$ Blood Transfusion Research Center, Mazandaran Blood Transfusion Center, Sari, Iran.

${ }^{9}$ Mazandaran Heart Center, Mazandaran

University of Medical Sciences, Iran.

*Email: s.azizi@mazums.ac.ir
}

\section{ABSTRACT}

BACKGROUND: Myocardial infarction (MI) as a term for a heart attack happens due to reduced blood flow to heart myocardium and lack of oxygen supply caused by plaques in the interior walls of coronary arteries. With respect to the importance of MI etiology, we aimed to study the relationship of MI and blood examination variables.

METHODS: This study was conducted in Mazandaran Heart Center as a hospital-based case-control Comprising 894 participants including 465 cases and 429 controls, individually matched by sex and age. Considered blood markers were analyzed using routine laboratory methods and equipment.

RESULTS: Of all participants, $64.3 \%$ of the cases and $51.0 \%$ of the controls were males with a mean age of $61.2( \pm 13.8)$ in cases and $62.4( \pm 14$.$) in controls. We could not find any$ differences between cases and controls for total cholesterol (TC), low-density lipoprotein (LDL), high-density lipoprotein $(H D L)$, and alkaline-phosphatase (ALP) $(P>0.05)$. However, levels of creatine-kinase-muscle/brain (CK-MB) $(P<0.0001)$, fasting-blood-sugar (FBS) $(P<0.0001)$ aspartateaminotransferase $(A S T) \quad(P<0.0001)$, alanine-transferase $(A L T)(P<0.0001)$ and erythrocyte sedimentation rate $(E S R)$ $(P=0.001)$ were significantly higher in cases compared to the controls $(P<0.05)$. Multivariable analyses revealed that the risk of MI was associated with high levels of AST (adjusted $O R=24.3,95 \% C I=3.5 \pm 165.6, P=0.001$ ) and $L D L$ (adjusted $O R=7.4,95 \% C I=1.0 \pm 51.8, P=0.001)$.

CONCLUSION: Our investigation indicated that the levels of CK-MB, FBS, AST, ALT and ESR were significantly higher in patients with MI. Besides, our findings showed that the risk of MI in cases with high levels of AST and LDL was about 24 and 7 times more than the control group respectively.

KEYWORDS: Cardiovascular Stroke, Heart Attack, Biochemical Markers, Serum Marker, Laboratory Markers 


\section{INTRODUCTION}

Myocardial infarction (MI) is defined as the presence of acute myocardial injury detected by abnormal cardiac biomarkers in the setting of evidence of acute myocardial ischemia (1). MI happens due to reduced blood flow to heart myocardium and lack of oxygen supply caused by plaques in the interior walls of coronary arteries (2). It is a major cause of death and disability worldwide (3). Over $15 \%$ of mortalities worldwide happens due to MI each year (4). According to the global burden of disease report 2017, cardiovascular diseases are the leading cause of death in Iran with 196.32 in $100 / 000$ people (5). There are approximately 3.6 million patients with cardiovascular disease in Iran, and $\mathrm{MI}$ is responsible for $46 \%$ deaths due to cardiovascular diseases (6).

In the clinical aspect, there is quite a number of enzymes, hormones, biological substances and other biomarkers derived from blood or urine that can be used in diagnosis (7). Since only myocardium contains a considerable amount of creatine kinasemuscle/brain (CK-MB), measuring CK-MB isoenzyme is a good indicator of MI (8). CK-MB appears 4 to 6 hours after the onset of chest pain, and it consists nearly $30 \%$ of CK in the myocardium (9). Plasma lipoproteins are risk factors for cardiovascular (CV) events after MI. High levels of low-density lipoprotein (LDL) is associated with increased risk of $\mathrm{CV}$ and high levels of high-density lipoprotein (HDL) associated with decreased risk of CV. Decreased LDL and variable HDL levels have been reported after MI and increased risk of inhospital mortality was associated with lower LDL levels (10).

Nonspecific tests which indicate inflammation can be used to diagnose various diseases. The inflammatory response following MI causes an increased erythrocyte sedimentation rate (ESR) (11). ESR is also a significant predictor of heart failure and might be useful for the diagnostic criterion for coronary heart disease (12). High levels of blood sugar can increase the risk of death and poor outcome in patients with MI, and it is associated with increased risk of in-hospital mortality in these patients (13). Fasting hyperglycemia in early MI could be a marker for high-risk individuals (14). Liver markers can also predict the outcome of patients after acute myocardial infarction. Increased serum level of alanine transferase (ALT) and aspartate aminotransferase (AST) are associated with a higher risk of CV events. ALT and AST can be elevated due to cardiac failure, and AST can be significantly correlated with death (15). Serum alkaline phosphatase (ALP) is also a good prognostic factor in MI patients (16).

Since clinical symptoms are not reliable enough in the diagnosis of MI and electrocardiography (ECG) can show uncertain patterns, serum biochemical markers can be helpful in confirming and diagnosis of MI (17). With respect to the importance of MI etiology using blood biomarkers, we aimed to investigate the relationship between MI and blood biomarkers in Mazandaran Heart Center.

\section{MATERIALS AND METHODS}

Study design: In this hospital-based case-control study, data were obtained from all patients admitted to the Mazandaran Heart Center in Sari city for the period of three months during students' internship in this center. This tertiary center is the largest heart center in the north of Iran, and most of the patients referred to this center are Mazandaranis. The study was conducted from September 2018 to December 2018 (according to the Persian solar calendar).

Study population: Hospitalized patients diagnosed with MI based on diagnostic criteria of the European Society of Cardiology (ESC) and the American College of Cardiology (ACC) guidelines (18) entered into our study as cases. Sex- and agematched ( \pm 1 years) seemingly healthy people, without a history of MI and negative troponin test participated in our study as controls. All of the participants were from the same geographic background (Mazandaranis). A consent letter was obtained from all participants, and no one refused to participate in the study. Exclusion criteria were as follows: 1) age under 18 years, 2) history of malignancy, 3) pregnant women, 4) insufficient medical record, and 5) participation refusal.

Laboratory measurements: After at least eight hours overnight fasting, blood samples were drawn from all participants. Serum levels of CK-MB, fasting blood sugar (FBS), total cholesterol (TC),

DOI: http://dx.doi.org/10.4314/ejhs.v30i1.2 
LDL, HDL, AST, ALT, and ALP were determined using the automatic biochemistry analyzer (Hitachi 912, Fameco, Japan). Troponin levels were analyzed using MINI VIDAS ${ }^{\circledR}$ immunoanalyzer (bioMérieux, France). According to Westergren method, whole blood of the patients was mixed with sodium citrate in a standardized calibrated tube, which are allowed to sit for 60 minutes in the LENA ESR analyzer to measure the sedimentation level.

Statistical analysis: The Statistical Package for the Social Sciences 16.0 (SPSS Inc., Chicago, Illinois, USA) was used for data analysis. A descriptive analysis of categorical variables was performed using frequencies and percentages. Variables comparisons were calculated using chi-square or Fisher exact tests, as appropriate. CK-MB $>190$, FBS $>115$, TC $>200$, LDL $>160$, HDL $<40$, AST $>35$, ALT $>40, A L P>306$ and ESR $>20$ were considered as abnormal levels. Comparisons of cases and controls regarding study variables were performed using the unpaired $t$-test or ANOVA for normally distributed parameters. In order to evaluate the interactive effects of study variables on MI occurrence, we used logistic regression model to calculate the odds ratio (OR) with $95 \%$ confidence interval $(95 \% \mathrm{CI})$. A $P$-value less than 0.05 was considered statistically significant.

This study was approved in the Ethical Review Committee of the Mazandaran University of Medical Science (MAZUMS) with ethical code IR.MAZUMS.REC.1398.469. The Student Research Committee of the MAZUMS sent a request to the Mazandaran Heart Center for collaboration. To comply with ethical standards, the consent form was filled out with all participants and their medical records were used confidentiality, exclusively for the research purpose.

\section{RESULTS}

In total, 894 subjects: 465 cases including 299 (64.3\%) males and 166(35.7\%) females and 429 controls including $219(51.0 \%)$ males and 210 $(49.0 \%)$ females, which matched by sex and age participated in the investigation. The characteristics of the cases and controls are presented in Table 1.

Table 1: Demographical and laboratory characteristics of the cases and controls

\begin{tabular}{lccc}
\hline Variable & Case $(\boldsymbol{n}=\mathbf{4 6 5})$ & Control $(\boldsymbol{n}=\mathbf{4 2 9})$ & $\boldsymbol{P}$-value \\
\hline Sex, male $n(\%)$ & $299(64.3)$ & $219(51.0)$ & matched \\
Age, years & $61.2 \pm 13.8$ & $62.4 \pm 14.1$ & matched \\
CK-MB, IU/L* & $108.3 \pm 155.6$ & $42.0 \pm 88.9$ & $<0.0001$ \\
FBS, mg/dL & $131.4 \pm 78.4$ & $101.5 \pm 44.2$ & $<0.0001$ \\
TC, $\mathrm{mg} / \mathrm{dL}^{*}$ & $174.5 \pm 49.7$ & $178.8 \pm 101.6$ & 0.340 \\
$\mathrm{LDL}, \mathrm{mg} / \mathrm{dL}^{*}$ & $150.6 \pm 768.8$ & $99.2 \pm 42.7$ & 0.103 \\
$\mathrm{HDL}, \mathrm{mg} / \mathrm{dL}^{*}$ & $36.6 \pm 12.1$ & $36.6 \pm 13.0$ & 0.754 \\
ESR, $\mathrm{mm} / \mathrm{h}^{*}$ & $47.0 \pm 48.7$ & $28.5 \pm 27.3$ & 0.001 \\
\hline
\end{tabular}

CK-MB: creatine kinase-muscle/brain; FBS: Fasting Blood Sugar; TC: Total Cholesterol; LDL: Low-Density Lipoprotein; HDL: High-Density Lipoprotein; AST: Aspartate Aminotransferase; ALT: Alanine transaminase; ALP: Alkaline phosphatase; ESR: Erythrocyte Sedimentation Rate. *mean \pm SD

The mean age of cases was 61.2 years $( \pm 13.8)$ while the mean age of controls was 62.4 years $( \pm 14.1)$. There were no differences between cases and controls regarding TC, LDL, HDL, and ALP.

As a cardiac marker, CK-MB levels were higher in MI patients in comparison to the control group $(P<0.0001)$. The mean amount of FBS was significantly higher in cases than in controls
$(P<0.0001)$. Regarding liver enzymes, although levels of ALP was not different between the two groups, levels of both AST $(P<0.0001)$ and ALT $(P<0.0001)$ were significantly higher in cases than controls. Moreover, level of ESR was meaningfully higher in cases $(P=0.001)$.

Multivariable analyses revealed that the increase in risk for MI was associated with high

DOI: http://dx.doi.org/10.4314/ejhs.v30i1.2 
levels of AST (adjusted $\mathrm{OR}=24.3$, significant relationships were observed for other $95 \% \mathrm{CI}=3.5 \pm 165.6, P=0.001$ ) and LDL (adjusted parameters. Risk estimates were present in Table 2.

$\mathrm{OR}=7.4,95 \% \mathrm{CI}=1.0 \pm 51.8, P=0.001)$. However, no

Table 2: Associations between considered variables and risk of Myocardial infarction

\begin{tabular}{lcc}
\hline Variable & OR $^{*}(\mathbf{9 5 \%} \mathbf{C I})$ & $\boldsymbol{P}$-value \\
\hline Sex & $0.050(0.008-0.308)$ & 0.001 \\
Age & $0.980(0.934-1.028)$ & 0.415 \\
CK-MB & $0.209(0.003-15.391)$ & 0.475 \\
FBS & $2.365(0.511-10.948)$ & 0.271 \\
TC & $0.566(0.107-2.990)$ & 0.503 \\
LDL & $7.481(1.080-51.806)$ & 0.042 \\
HDL & $3.629(0.757-17.396)$ & 0.107 \\
AST & $24.366(3.583-165.697)$ & 0.001 \\
ALT & $0.791(0.063-9.879)$ & 0.856 \\
ALP & $1.857(0.376-9.166)$ & 0.447 \\
ESR & $0.263(0.043-1.616)$ & 0.149 \\
\hline
\end{tabular}

CK-MB: creatine kinase-muscle/brain; FBS: Fasting Blood Sugar; TC: Total Cholesterol; LDL: Low-Density Lipoprotein; HDL: High-Density Lipoprotein; AST: Aspartate Aminotransferase; ALT: Alanine transaminase; ALP: Alkaline phosphatase; ESR: Erythrocyte Sedimentation Rate.

*Adjusted by age and sex

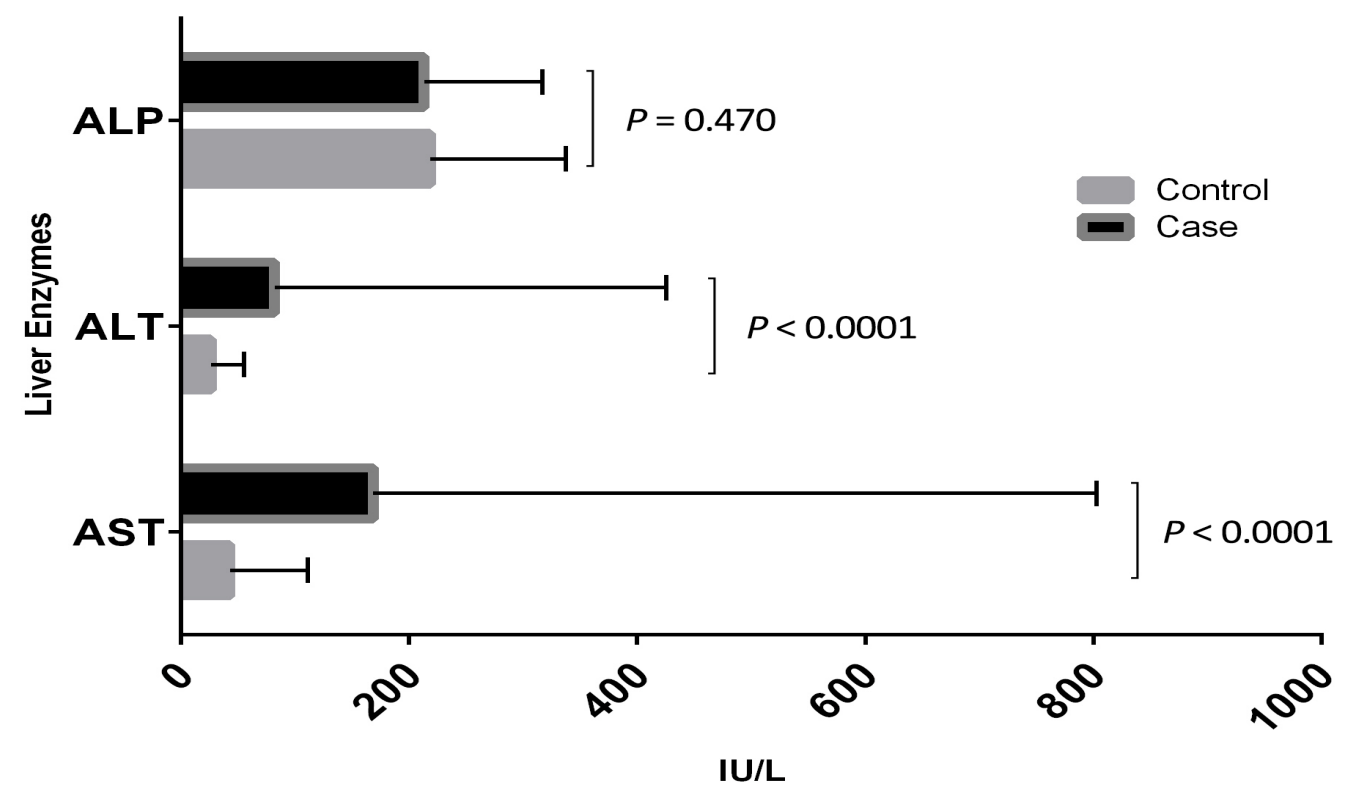

Figure 1: Differences between cases and controls regarding liver enzymes

\section{DISCUSSION}

Our investigation indicated that levels of CK-MB, FBS, AST, ALT, and ESR were significantly higher in patients with MI. Besides, our findings showed that the risk of MI in cases with high levels of AST and LDL was about 24 and 7 times more than the control group respectively.

DOI: http://dx.doi.org/10.4314/ejhs.v30i1.2 
Our findings showed that CK-MB levels were significantly higher in cases with MI, and many other studies reported the good performance of this biomarker in detection of MI $(19,20)$. In contrast, it is also reported that the weak sensitivity and specificity for CK-MB in this regard and its accuracy is still controversial, especially after resuscitation (21). However, it seems that it can be a useful marker along with other diagnostic approaches for MI detection.

Regarding FBS, our results were in the same line with the study of Abbasi et al., which considered the high levels of FBS as one of the MI risk factors (22). It is remarkable that the study of Ishihara et al. showed that hyperglycemia is associated with MI and that it can deteriorate the prognosis in both diabetic and non-diabetic individuals (23). It is also reported that hyperglycemia is associated with the development of kidney injury and left ventricular dysfunction (because of the no-reflow phenomenon) in patients with MI (24, 25). Hyperglycemia can affect MI through several ways such as induction of electrophysiological alterations following arrhythmias, ischemic preconditioning, coagulation alteration, which likely to increase thrombosis activation, inflammatory amplification, and inducing endothelial dysfunction (26).

Among liver enzymes, levels of AST and ALT were elevated in MI patients significantly in comparison to the control group, which was also reported in the study of Lofthus et al. (27). About ALP, although we did not find any relationships with MI, Nunes et al. found that high levels of ALP can act as a diagnostic factor for decreased survival rate and renal function in male diabetic patients with MI (28).

Besides, our check results showed that the probability of MI in individuals with high levels of AST is nearly 17 times more than the control group. This enzyme was one of the first cardiac biomarkers used in the year 1954, and because of weak specificity for the matter, it is no longer used as a gold standard. Thereafter, total creatine kinase level and lactate dehydrogenase were used as a diagnostic factor for MI by the years 1959 and 1960 respectively $(29,30)$. Eventually, the World Health Organization (WHO) suggested using these three factors for MI diagnosis by the year 1979, which was altered by the immunoassay's development in $1980(31,32)$.

Regarding lipids profile, our findings showed that cases with high levels of LDL are at risk of MI 7 times more than the control group, which is in the same line with previous studies $(33,34)$. However other members of lipids, although we could not find any relationships, previous studies indicated a relationship between low levels of HDL (35) and high levels of triglycerides (36) and risk of MI. However, facts regarding HDL are still controversial (37).

Our investigation indicated that high levels of ESR could be related to poor prognosis of MI, which was previously reported in several studies similarly $(38,39)$. Therefore, it seems that ESR can be considered as a prognostic factor for monitoring of patients with MI to prevent the heart acute attacks (40).

As a strength of the study, to our knowledge, there is no investigation regarding the association of blood biomarkers with MI in this extent, at least in Iran. However, due to the retrospective nature of the study as a limitation, it is recommended that a comprehensive longitudinal cohort study be designed in order to examine the accurate role of these markers in MI.

In conclusion, we found that levels of CK-MB, FBS, AST, ALT, and ESR were significantly higher in patients with MI compared to the control group. Besides, our results indicated that individuals with high levels of AST were at risk of MI about 24 times more than the control group. Moreover, this risk was also 7 times higher in cases with high levels of LDL. Hence, clinicians may consider these factors as a valuable diagnostic or prognostic factor for patients with MI to prevent the next attacks and for a healthy population to prevent the first attack. Nevertheless, more comprehensive studies in different regions need to be done to avoid the geographical and race biases, which may affect the matter. 


\section{ACKNOWLEDGMENTS}

The authors would like to express their gratitude to the Student Research Committee of Mazandaran University of Medical Sciences for supporting this project with the code 4783 on March 5, 2019. We also appreciate the cooperation of staff working at Mazandaran Heart Center.

\section{REFERENCES}

1. Thygesen K, Alpert JS, Jaffe AS, Chaitman BR, Bax JJ, Morrow DA, White HD. Fourth universal definition of myocardial infarction. $\mathrm{J}$ Am Coll Cardiol. 2018;72(18):2231-64.

2. Lu L, Liu M, Sun R, Zheng Y, Zhang P. Myocardial Infarction: Symptoms and Treatments. Cell Biochem Biophys. 2015;72(3):865-7.

3. Thygesen K, Alpert JS, Jaffe AS, Simoons ML, Chaitman BR, White HD. Third universal definition of myocardial infarction. Circulation. 2012;126(16):2020-35.

4. Jayaraj JC, Davatyan K, Subramanian S, Priya J. Epidemiology of Myocardial Infarction. Myocardial Infarction: IntechOpen; 2018.

5. Bashzar S, Tourani S, Nikfar S, Ravaghi H, Habibi M. Health Technology Assessment of TNK-ase vs. Reteplase in the Treatment of Acute Myocardial Infarction in Iran. Health-Based Research. 2018;4(1):51-62.

6. Taghipour B, Froelicher ES, Goudarzian AH, Chan YH, Nia HS, Yaghoobzadeh A, Haghdoost AA. Clinical Manifestation of Acute Myocardial Infarction: Classified by Age and Gender. J Cri Care Nurs. 2018;11(1).

7. Braunwald E. Biomarkers in heart failure. N Engl J Med. 2008;358(20):2148-59.

8. Navin TR, Hager WD. Creatine kinase MB isoenzyme in the evaluation of myocardial infarction. Curr Probl Cardiol. 1979;3(12):1-32.

9. Jacob R, Khan M. Cardiac Biomarkers: What Is and What Can Be. Indian Journal of Cardiovascular Disease in Women WINCARS. 2018;3(04):240-4.

10. Reddy VS, Bui QT, Jacobs JR, Begelman SM, Miller DP, French WJ. Relationship Between Serum Low-Density Lipoprotein Cholesterol and In-hospital Mortality Following Acute
Myocardial Infarction (The Lipid Paradox). Am J Cardiol. 2015;115(5):557-62.

11. Boltax AJ, Fischel EE. Serologic tests for inflammation: Serum complement, C-reactive protein and erythrocyte sedimentation rate in myocardial infarction. $\mathrm{Am} J \mathrm{Med}$. 1956;20(3):418-27.

12. Yayan J. Erythrocyte sedimentation rate as a marker for coronary heart disease. Vasc Health Risk Manag. 2012;8:219-23.

13. Capes SE, Hunt D, Malmberg K, Gerstein HC. Stress hyperglycaemia and increased risk of death after myocardial infarction in patients with and without diabetes: a systematic overview. The Lancet. 2000;355(9206):773-8.

14. Qin Y, Yan G, Luo E, Ma C, Liu JJA. Effects of Hyperglycemia on the In-hospital and Long-term Prognosis of Patients with Acute ST-segment Elevation Myocardial Infarction. Angiology. 2018;6(211):2.

15. Baars T, Sowa JP, Neumann U, Hendricks S, Jinawy M, Kälsch J, Gerken G, Rassaf T, Heider D, Canbay A. Liver parameters as part of a non-invasive model for prediction of allcause mortality after myocardial infarction. Arch Med Sci. 2018;14(1).

16. Yanishi K, Nakamura T, Nakanishi N, Yokota I, Zen K, Yamano T, et al. A Simple Risk Stratification Model for ST-Elevation Myocardial Infarction (STEMI) from the Combination of Blood Examination Variables: Acute Myocardial InfarctionKyoto Multi-Center Risk Study Group. PloS one. 2016;11(11):e0166391.

17. Nigam PK. Biochemical markers of myocardial injury. Indian J Clin Biochem. 2007;22(1):10-7.

18. Thygesen K, Alpert JS, Jaffe AS, Chaitman BR, Bax JJ, Morrow DA, et al. Fourth universal definition of myocardial infarction. Eur Heart J. 2018;40(3):237-69.

19. Zimmerman J, Fromm R, Meyer D, Boudreaux A, Wun CC, Smalling R, et al. Diagnostic marker cooperative study for the diagnosis of myocardial infarction. Circulation. 1999;99(13):1671-7.

20. Laurino JP, Bender EW, Kessimian N, Chang $\mathrm{J}$, Pelletier T, Usategui M. Comparative sensitivities and specificities of the mass

DOI: http://dx.doi.org/10.4314/ejhs.v30i1.2 
measurements of CK-MB2, CK-MB, and myoglobin for diagnosing acute myocardial infarction. Clin Chem. 1996;42(9):1454-9.

21. Kruse JM, Enghard P, Schroder T, Hasper D, Kuhnle Y, Jorres A, et al. Weak diagnostic performance of troponin, creatine kinase and creatine kinase-MB to diagnose or exclude myocardial infarction after successful resuscitation. Int J Cardiol. 2014;173(2):21621.

22. Abbasi A, Saleem A, Rather A, Arooj S, Habib N, Aziz W. Statistical study of the risk factors of myocardial infarction in the patients of district Muzaffarabad capital of Azad Jammu and Kashmir. Pak J Pharm Sci. 2015;28(3):921-6.

23. Ishihara M, Kojima S, Sakamoto T, Asada Y, Tei C, Kimura K, et al. Acute hyperglycemia is associated with adverse outcome after acute myocardial infarction in the coronary intervention era. Am Heart $J$. 2005;150(4):814-20.

24. Moriyama $\mathrm{N}$, Ishihara $\mathrm{M}$, Noguchi $\mathrm{T}$, Nakanishi M, Arakawa T, Asaumi Y, et al. Admission hyperglycemia is an independent predictor of acute kidney injury in patients with acute myocardial infarction. Circ $J$. 2014;78(6):1475-80.

25. Kosuge M, Kimura K, Ishikawa T, Shimizi T, Hibi K, Toda N, et al. Persistent hyperglycemia is associated with left ventricular dysfunction in patients with acute myocardial infarction. Circ J. 2005;69(1):238.

26. Ceriello A. Acute hyperglycaemia: a 'new' risk factor during myocardial infarction. Eur Heart J. 2005;26(4):328-31.

27. Lofthus DM, Stevens SR, Armstrong PW, Granger CB, Mahaffey KW. Pattern of liver enzyme elevations in acute ST-elevation myocardial infarction. Coron Artery Dis. 2012;23(1):22-30.

28. Nunes JP, Melao F, Godinho AR, Rodrigues JD, Maciel MJ. Plasma alkaline phosphatase and survival in diabetic patients with acute myocardial infarction. Ann Transl Med. 2016;4(11):210.
29. Ladenson JH. A personal history of markers of myocyte injury [myocardial infarction]. Clin Chim Acta. 2007;381(1):3-8.

30. Dolci A, Panteghini M. The exciting story of cardiac biomarkers: from retrospective detection to gold diagnostic standard for acute myocardial infarction and more. Clin Chim Acta. 2006;369(2):179-87.

31. Nomenclature and criteria for diagnosis of ischemic heart disease. Report of the Joint International Society and Federation of Cardiology/World Health Organization task force on standardization of clinical nomenclature. Circulation. 1979;59(3):607-9.

32. Chan D, Ng LLJBM. Biomarkers in acute myocardial infarction. BMC Med. 2010;8(1):34.

33. Xenophontos $\mathrm{S}$, Hadjivassiliou $\mathrm{M}$, Karagrigoriou A, Demetriou N, Miltiadous G, Marcou I, et al. Low HDL cholesterol, smoking and IL-13 R130Q polymorphism are associated with myocardial infarction in Greek Cypriot males. A pilot study. Open Cardiovasc Med J. 2008;2:52-9.

34. Tonelli M, Muntner P, Lloyd A, Manns B, Klarenbach S, Pannu N, et al. Association between LDL-C and risk of myocardial infarction in CKD. $J$ Am Soc Nephrol. 2013;24(6):979-86.

35. Ramirez A, Hu PP. Low High-Density Lipoprotein and Risk of Myocardial Infarction. Clin Med Insights Cardiol. 2015;9:113-7.

36. Nordestgaard BG, Benn M, Schnohr P, Tybjaerg-Hansen A. Nonfasting triglycerides and risk of myocardial infarction, ischemic heart disease, and death in men and women. JAMA. 2007;298(3):299-308.

37. Voight BF, Peloso GM, Orho-Melander M, Frikke-Schmidt R, Barbalic M, Jensen MK, et al. Plasma HDL cholesterol and risk of myocardial infarction: a mendelian randomisation study. The Lancet. 2012;380(9841):572-80.

38. Erikssen G, Liestol $\mathrm{K}$, Bjornholt JV, Stormorken H, Thaulow E, Erikssen J. Erythrocyte sedimentation rate: a possible marker of atherosclerosis and a strong

DOI: http://dx.doi.org/10.4314/ejhs.v30i1.2 
predictor of coronary heart disease mortality. Eur Heart J. 2000;21(19):1614-20.

39. Andresdottir MB, Sigfusson N, Sigvaldason $\mathrm{H}$, Gudnason V. Erythrocyte sedimentation rate, an independent predictor of coronary heart disease in men and women: The Reykjavik Study. Am $J$ Epidemiol. 2003;158(9):844-51.
40. Fatih Ozlu M, Sen N, Fatih Karakas M, Turak O, Ozcan F, Kanat S, et al. Erythrocyte sedimentation rate in acute myocardial infarction as a predictor of poor prognosis and impaired reperfusion. Med Glas (Zenica). 2012;9(2):189-97.

DOI: http://dx.doi.org/10.4314/ejhs.v30i1.2 